\title{
Further Results on Reachable Set Bounding for Discrete-Time System with Time-Varying Delay and Bounded Disturbance Inputs
}

\author{
Wei Kang $\left(\mathbb{D},{ }^{1,2}\right.$ Hao Chen, ${ }^{3}$ Kaibo Shi $\mathbb{D}^{4},{ }^{4}$ and Jun Cheng $\mathbb{D}^{5}$ \\ ${ }^{1}$ School of Information Engineering, Fuyang Normal University, Fuyang 236041, China \\ ${ }^{2}$ School of Mathematical Sciences, University of Electronic Science and Technology of China, Chengdu 611731, China \\ ${ }^{3}$ School of Mathematics Science, Huaibei Normal University, Huaibei 235000, China \\ ${ }^{4}$ School of Information Science and Engineering, Chengdu University, Chengdu 610106, China \\ ${ }^{5}$ School of Science, Hubei University for Nationalities, Enshi, Hubei 445000, China
}

Correspondence should be addressed to Wei Kang; kangwei0830@163.com

Received 16 November 2017; Accepted 20 February 2018; Published 28 March 2018

Academic Editor: Eulalia Martínez

Copyright (C) 2018 Wei Kang et al. This is an open access article distributed under the Creative Commons Attribution License, which permits unrestricted use, distribution, and reproduction in any medium, provided the original work is properly cited.

\begin{abstract}
This paper investigates the problem of reachable set bounding for discrete-time system with time-varying delay and bounded disturbance inputs. Together with a new Lyapunov-Krasovskii functional, discrete Wirtinger-based inequality, and reciprocally convex approach, sufficient conditions are derived to find an ellipsoid to bound the reachable sets of discrete-time delayed system. The main advantage of this paper lies in two aspects: first, the initial state vectors are not necessarily zero; second, the obtained criteria in this paper do not really require all the symmetric matrices involved in the employed Lyapunov-Krasovskii functional to be positive definite. Finally, two numerical examples are provided to illustrate the effectiveness of the proposed method.
\end{abstract}

\section{Introduction}

The reachable set of dynamic system is defined as a collection of system state vectors in presence of all admissible input disturbances. As is known to all, the phenomenon of disturbance inputs is usually unavoidable in engineering and practical systems because of data transformation, measurement errors, linearisation approximations, and some unknown disturbances. Therefore, the reachable set bounding for dynamic systems with input disturbances is an important and challenging research topic in robust control theory and practical engineering. Then, applications of reachable set bounding can be found in various fields such as peak-topeak minimization, aircraft collision avoidance, parameter estimation, and constrained control design [1-3]. On the other hand, time delay often occurs in various real-world systems and the existence of it may lead to instability, oscillation, or bad system performance [4-6]. Thus, stability and control of time-delay systems plays an important role in the field of engineering. As a result, many scholars have devoted themselves to the study of time-delay systems [718] and varieties of methods have been introduced to solve the stability analysis and control synthesis of delayed systems, such as delay-partitioning method $[10,11]$, reciprocally convex approach [13], and the free-weighting matrices technique [14].

In many practical applications, we often require to find a bound of a set of all the states that are reachable from a given set. Two definitions on forward and backward reachable sets are introduced in [18-20]. Those notions have been widely applied to state bounding observers, safety verification, and model checking. Forward reachable set with regard to a given initial set of a dynamic system is the set of all the states starting from the given initial set. It should be pointed out that reachable set defined in [21-25] is a special case of forward reachable set when the given initial set contains only the origin point. Most of existing results about forward reachable set bounding were reported for dynamic systems without time delay. As for the reachable set bounding problem of timedelay systems, it was firstly solved by Fridman and Shaked 
in [2] based on Lyapunov-Razumikhin approach. Then, some excellent achievements on reachable set bounding for dynamical systems have been proposed in the literature [2131]. By using an enlarging Lyapunov-Krasovskii functional and delay-partitioning method, an improved condition was provided in [21]. Zuo et al. introduced the maximal Lyapunov functional method to deal with the problem of reachable set bounding for linear systems in [22]. Subsequently, the maximal Lyapunov functional approach has been adopted to research the reachable set estimation for delayed neural network [23]. In [25], the authors studied the problem of reachable set estimation and synthesis for delayed systems by using delay-decomposition technique and reciprocally convex approach. Recently, the problem of reachable set estimation for delayed singular systems was considered in [31] based on Lyapunov method. In [27], new explicit delayindependent conditions are obtained by utilizing a novel method which does not involve the Lyapunov-Krasovskii functional way. In [32], the authors presented a new approach to obtain the smallest box which bounds all reachable sets of a class of nonlinear system. The problem of state bounding for homogeneous positive nonlinear systems was studied in [33]. In this paper, we will further study the forward reachable set bounding problem for delayed system.

It is worth noting that all the above-mentioned research has been focused on continuous-time systems. However, with the progress of computer-based computational techniques, discrete-time systems play a considerable role in many practical applications. Therefore, the stability and control of discrete-time delayed systems is an important issue and has been studied by many researchers [34-40]. Up to now, there are few papers considering the reachable set estimation of discrete-time systems with time-varying delay. In [38], an ellipsoidal-like bounding of the reachable set for discrete-time system with time-varying delay is shown by using the delay-decomposition and the free-weighting matrix techniques. In [39], the problem of reachable set bounding for discrete-time polytopic systems with multiple constant delays was studied; delay-dependent conditions were derived in the form of relaxed matrix inequalities. Compared with the results in $[38,39]$, some less conservative conditions are obtained in [40] by employing a novel Lyapunov-Krasovskii functional combining with delay-decomposition technique and reciprocally convex method. In [41], the reachable set estimation of delayed discrete-time T-S fuzzy systems was considered. The reachable set estimation problem for discrete-time Markovian jump neural networks was firstly investigated in [42]. However, there is still much room to investigate the problem of forward reachable set bounding for discrete-time system with time-varying delay, which is our motivation.

In this paper, we concentrate on the forward reachable set bounding problem for discrete-time system with timevarying delay. The ellipsoidal reachable set estimation conditions of discrete-time linear systems are obtained by using discrete Wirtinger-based inequality and reciprocally convex approach. The novelty of this paper is three aspects: first, a relaxed Lyapunov-Krasovskii functional, which does not require all the involved symmetric matrices to be positive definite, is employed to solve the addressed problem; second, the initial state vectors are not necessarily zero, which is more general than the existing results $[38,39]$; third, discrete Wirtinger-based inequality is taken into account in this paper to deal with the problem of reachable set bounding for delayed discrete-time systems. Finally, two numerical examples are provided to validate the effectiveness of the proposed method.

Notations. Throughout this paper, $R>0(R \geqslant 0, R<$ $0, R \leqslant 0$ ) means that the matrix $R$ is positive definite (positive semidefinite, negative definite, and negative semidefinite); $\mathbb{R}^{m \times n}$ is the set of $m \times n$ real matrices; the superscripts -1 and $T$ denote the inverse and transpose of a matrix, respectively; $*$ denotes the symmetric block in symmetric matrix; $I$ denotes the identity matrix with compatible dimensions; $N$ denotes the set of natural number; and $\mathscr{Z}$ denotes the set of integers.

\section{Problem Statement and Preliminaries}

We consider the following discrete-time system with timevarying delay:

$$
\begin{array}{r}
x(k+1)=A_{1} x(k)+A_{2} x(k-\tau(k))+B \omega(k), \\
x(\theta)=\varphi(\theta), \quad \theta \in\left\{-\tau_{M},-\tau_{M}+1, \ldots, 0\right\},
\end{array}
$$

where $x(k)=\left[x_{1}(k), x_{2}(k), \ldots, x_{n}(k)\right]^{T} \in \mathbb{R}^{n}$ is the state vector of the system, $A_{1}, A_{2}$, and $B$ are known constant matrices with appropriate dimensions, $\tau(k)$ is the timevarying delay and satisfies $0<\tau_{m} \leq \tau(k) \leq \tau_{M}$, and $\omega(k) \in \mathbb{R}^{l}$ represents a disturbance which satisfies

$$
\omega^{T}(k) \omega(k) \leq \omega_{d}^{2} .
$$

$\varphi(\theta)$ is the initial condition function satisfying

$$
\varphi^{T}(\theta) \varphi(\theta) \leq \sigma_{1}, \quad \theta \in\left\{-\tau_{M},-\tau_{M}+1, \ldots, 0\right\} .
$$

Define that $\eta(k)=x(k+1)-x(k)$, which satisfies

$$
\eta^{T}(k) \eta(k) \leq \sigma_{2}, \quad k \in\left\{-\tau_{M},-\tau_{M}+1, \ldots, 0\right\} .
$$

In this paper, we denote reachable set of system (1) as

$$
\mathscr{R}=\{x(k) \mid x(k), \omega(k) \text { satisfy }(1)-(4), k \geq 0\} .
$$

Our aim is to find an ellipsoid to bound the reachable set $\mathscr{R}$ of system (1).

In addition, we give some lemmas which will be used in deriving our results.

Lemma 1. Let $V(k)$ be a positive-definite function with $\omega(k)$ satisfying $\omega^{T}(k) \omega(k) \leq \omega_{d}^{2}$; if there exists a scalar $0<\beta<1$, such that

$$
\Delta V(k)+(1-\beta) V(k)-\frac{1-\beta}{\omega_{d}^{2}} \omega^{T}(k) \omega(k) \leq 0,
$$

then, we have $V(k) \leq \max \{1, V(0)\}, \forall k \in N$. 
Proof. From (6), we can get

$$
\begin{aligned}
& V(k+1)-V(k)+(1-\beta) V(k)-\frac{1-\beta}{\omega_{d}^{2}} \omega^{T}(k) \omega(k) \\
& \quad \leq 0
\end{aligned}
$$

then, we have

$$
\begin{aligned}
V(k+1) & \leq \beta V(k)+\frac{1-\beta}{\omega_{d}^{2}} \omega^{T}(k) \omega(k) \\
& \leq \beta V(k)+(1-\beta) \\
& \leq \beta^{2} V(k-1)+\left(1-\beta^{2}\right) \\
& \vdots \\
& \leq \beta^{k+1} V(0)+\left(1-\beta^{k+1}\right)
\end{aligned}
$$

then, we can get

$$
V(k) \leq 1+\beta^{k}(V(0)-1) \leq \max \{1, V(0)\}, \quad \forall k \geq 0 . \quad \text { (9) }
$$

This completes the proof.
Lemma 2 (see [18]). For a given matrix $Y>0$, and three given nonnegative integers $a, b, k$ satisfying $a \leq b \leq k$, vector function $x(i):[k-b, k-a] \cap \mathscr{Z} \rightarrow \mathbb{R}^{n}, \eta(i)=x(i+1)-x(i)$, we denote

$$
\begin{aligned}
& \omega(k, a, b) \\
& = \begin{cases}\frac{1}{b-a}\left[2 \sum_{i=k-b}^{k-a-1} x(i)+x(k-a)-x(k-b)\right], & a<b, \\
2 x(k-a), & a=b ;\end{cases}
\end{aligned}
$$

then, we have

$$
\begin{aligned}
- & (b-a) \sum_{i=k-b}^{k-a-1} \eta^{T}(i) Y \eta(i) \\
\leq & -[x(k-a)-x(k-b)]^{T} Y[x(k-a)-x(k-b)] \\
& -3 \Lambda^{T} Y \Lambda
\end{aligned}
$$

where $\Lambda=x(k-a)+x(k-b)-\omega(k, a, b)$.

Lemma 3 (see [13]). Let $f_{1}, f_{2}, \ldots, f_{n}: \mathscr{R}^{m} \mapsto \mathbb{R}$ have positive values in an open subset $D$ and $\mathbb{R}^{m}$; then, the reciprocally convex combination of $f_{i}$ over $D$ satisfies

$$
\begin{aligned}
\min _{\left\{\alpha_{i} \mid \alpha_{i}>0 \sum_{i} \alpha_{i}=1\right\}} & \sum_{i} \frac{1}{\alpha_{i}} f_{i}(t)=\sum_{i} f_{i}(t)+\max _{g_{i, j}(t)} \sum_{i \neq j} g_{i j}(t), \\
\text { subject to } & \left\{g_{i, j} \mathbb{R}^{m} \rightarrow \mathbb{R}, g_{j, i}(t)=g_{i, j}(t),\left[\begin{array}{cc}
f_{i}(t) & g_{i, j}(t) \\
g_{j, i}(t) & f_{j}(t)
\end{array}\right] \geq 0\right\} .
\end{aligned}
$$

\section{Main Results}

Before main results, we define block entry matrices as $e_{i} \in \mathbb{R}^{9 n \times n}(i=1,2, \ldots, 9)$ (e.g., $\left.e_{5}=[0,0,0,0, I, 0,0,0,0]^{T}\right)$. The other vectors are defined as

$$
\begin{aligned}
& \kappa_{1}(k)=\omega\left(k, 0, \tau_{m}\right)= \begin{cases}\frac{1}{\tau_{m}}\left[2 \sum_{i=k-\tau_{m}}^{k-1} x(i)+x(k)-x\left(k-\tau_{m}\right)\right], & \tau_{m}>0 \\
2 x(k), & \tau_{m}=0\end{cases} \\
& \kappa_{2}(k)=\omega\left(k, \tau_{m}, \tau(k)\right)= \begin{cases}\frac{1}{\tau(k)-\tau_{m}}\left[2 \sum_{i=k-\tau(k)}^{k-\tau_{m}-1} x(i)+x\left(k-\tau_{m}\right)-x(k-\tau(k))\right], & \tau_{m}<\tau(k), \\
2 x\left(k-\tau_{m}\right), & \tau_{m}=\tau(k),\end{cases} \\
& \kappa_{3}(k)=\omega\left(k, \tau(k), \tau_{M}\right)= \begin{cases}\frac{1}{\tau_{M}-\tau(k)}\left[2 \sum_{i=k-\tau_{M}}^{k-\tau(k)-1} x(i)+x(k-\tau(k))-x\left(k-\tau_{M}\right)\right], & \tau(k)<\tau_{M}, \\
2 x(k-\tau(k)), & \tau_{M}=\tau(k) .\end{cases}
\end{aligned}
$$


Theorem 4. For given scalar $0<\beta<1$, if there exist positive scalars $\lambda_{1}, \lambda_{2}, \lambda_{3}, \lambda_{4}, \lambda_{5}$, symmetric matrices $P_{1} \in \mathbb{R}^{n \times n}, Q_{1} \in$ $\mathbb{R}^{n \times n}, Q_{2} \in \mathbb{R}^{n \times n}, Z_{1} \in \mathbb{R}^{n \times n}>0, Z_{2} \in \mathbb{R}^{n \times n}>0, W \in$ $\mathbb{R}^{n \times n}>0$, and matrices $S \in \mathbb{R}^{2 n \times 2 n}, M_{1} \in \mathbb{R}^{n \times n}, M_{2} \in \mathbb{R}^{n \times n}$, $M_{3} \in \mathbb{R}^{n \times n}$, such that

$$
\begin{aligned}
\Phi_{1} & =\left[\begin{array}{ll}
\overline{Z_{2}} & S \\
* & \overline{Z_{2}}
\end{array}\right]>0, \\
\Omega_{1} & >0, \\
\Omega_{2} & >0, \\
P_{1} & \leq \lambda_{1} I, \\
Q_{1} & \leq \lambda_{2} I, \\
Q_{2} & \leq \lambda_{3} I, \\
0 & <Z_{1} \leq \lambda_{4} I, \\
0 & <Z_{2} \leq \lambda_{5} I, \\
\mu_{1} \sigma_{1}+\mu_{2} \sigma_{2} & \leq 1, \\
\Xi & =\Xi_{1}+\Xi_{2}<0,
\end{aligned}
$$

then, the reachable set of system (1) is contained in the following ellipsoid:

$$
\varepsilon(W)=\left\{\xi \in \mathbb{R}^{n} \mid \xi^{T} W \xi \leq 1\right\}
$$

where

$$
\begin{aligned}
\Omega_{1} & =\left[\begin{array}{cc}
\delta P_{1}+Z_{1}-\delta W & -Z_{1} \\
* & Q_{1}+Q_{2}+Z_{1}
\end{array}\right], \\
\Omega_{2} & =\left[\begin{array}{cc}
\delta P_{1}+\frac{\tau_{M m}}{\tau_{M}} Z_{2}-\delta W & -\frac{\tau_{M m}}{\tau_{M}} Z_{2} \\
* & Q_{2}+\frac{\tau_{M m}}{\tau_{M}} Z_{2}
\end{array}\right], \\
\Xi_{1} & =\left(e_{1}+e_{8}\right) P_{1}\left(e_{1}+e_{8}\right)^{T}-\beta e_{1} P_{1} e_{1}+e_{1} Q_{1} e_{1}^{T} \\
& +e_{1} Q_{2} e_{1}^{T}-\beta^{\tau_{m}} e_{2} Q_{1} e_{2}^{T}-\beta^{\tau_{M}} e_{4} Q_{2} e_{4}^{T}+\tau_{m}^{2} e_{8} Z_{1}^{T} e_{8} \\
& +\tau_{M m}^{2} e_{8} Z_{2}^{T} e_{8}-\frac{1}{\omega_{d}^{2}} e_{9} e_{9}^{T}-\beta^{\tau_{m}} \Lambda_{1}\left[\begin{array}{cc}
Z_{1} & 0 \\
0 & 3 Z_{1}
\end{array}\right] \Lambda_{1}^{T} \\
& -\beta^{\tau_{M}} \Lambda_{2}\left[\begin{array}{cc}
Z_{2} & S \\
* & \overline{Z_{2}}
\end{array}\right] \Lambda_{2}^{T}, \\
\Lambda_{1} & =\left[e_{1}-e_{2}, e_{1}+e_{2}-e_{5}\right], \\
\Lambda_{2} & =\left[e_{2}-e_{3}, e_{2}+e_{3}-e_{6}, e_{3}-e_{4}, e_{3}+e_{4}-e_{7}\right], \\
\Xi_{2} & =2\left[e_{1} M_{1}+e_{3} M_{2}+e_{8} M_{3}\right] \\
& \cdot\left[e_{1}(A-I)^{T}+e_{3} B^{T}-e_{8}\right]^{T},
\end{aligned}
$$

$$
\begin{aligned}
& \overline{Z_{2}}=\left[\begin{array}{cc}
Z_{2} & 0 \\
0 & 3 Z_{2}
\end{array}\right], \\
& \mu_{1}=\left|\lambda_{1}\right|+\tau_{m}\left|\lambda_{2}\right|+\tau_{M}\left|\lambda_{3}\right|, \\
& \mu_{2}=\frac{\tau_{m}\left(\tau_{m}-1\right)}{2} \lambda_{4}+\beta^{\tau_{m}} \frac{\tau_{M m}\left(\tau_{M}+\tau_{m}+1\right)}{2} \lambda_{5}, \\
& \tau_{M m}=\tau_{M}-\tau_{m}, \\
& \delta=\frac{1-\beta}{1-\beta^{\tau_{M}}} .
\end{aligned}
$$

Proof. We construct the following Lyapunov-Krasovskii functional for system (1):

$$
V(k)=\sum_{i=1}^{3} V_{i}(k),
$$

where

$$
\begin{aligned}
V_{1}(k)= & x^{T}(k) P_{1} x(k), \\
V_{2}(k)= & \sum_{i=k-\tau_{m}}^{k-1} \beta^{k-1-i} x^{T}(i) Q_{1} x(i) \\
& +\sum_{i=k-\tau_{M}}^{k-1} \beta^{k-1-i} x^{T}(i) Q_{2} x(i), \\
V_{3}(k)= & \tau_{m} \sum_{j=-\tau_{m}}^{-1} \sum_{i=k+j}^{k-1} \beta^{k-1-i} \eta^{T}(i) Z_{1} \eta(i) \\
& +\tau_{M m} \sum_{j=-\tau_{M}}^{-\tau_{m}-1} \sum_{i=k+j}^{k-1} \beta^{k-1-i} \eta^{T}(i) Z_{2} \eta(i) .
\end{aligned}
$$

First of all, the functional $V(k)$ is required to be positive definite. By using Jensen inequality [37], the following can be obtained:

$$
\begin{gathered}
\tau_{m} \sum_{j=-\tau_{m}}^{-1} \sum_{i=k+j}^{k-1} \beta^{k-1-i} \eta^{T}(i) Z_{1} \eta(i) \geq \tau_{m} \sum_{j=-\tau_{m}}^{-1} \beta^{-j-1} \frac{1}{-j} \\
\cdot\left(\sum_{i=k+j}^{k-1} \eta(i)\right)^{T} Z_{1}\left(\sum_{i=k+j}^{k-1} \eta^{T}(i)\right) \\
\geq \sum_{j=-\tau_{m}}^{-1} \beta^{-j-1}\left(\begin{array}{c}
x(k) \\
x(k+j)
\end{array}\right)^{T} \\
\cdot\left[\begin{array}{cc}
Z_{1} & -Z_{1} \\
* & Z_{1}
\end{array}\right]\left(\begin{array}{c}
x(k) \\
x(k+j)
\end{array}\right) \\
\geq \sum_{i=k-\tau_{m}}^{k-1} \beta^{k-i-1}\left(\begin{array}{c}
x(k) \\
x(i)
\end{array}\right)^{T}\left[\begin{array}{cc}
Z_{1} & -Z_{1} \\
* & Z_{1}
\end{array}\right]\left(\begin{array}{c}
x(k) \\
x(i)
\end{array}\right)
\end{gathered}
$$




$$
\begin{aligned}
\tau_{M m} & \sum_{j=-\tau_{M}}^{-\tau_{m}-1} \sum_{i=k+j}^{k-1} \beta^{k-1-i} \eta^{T}(i) Z_{2} \eta(i) \\
\geq & \tau_{M m} \sum_{j=-\tau_{M}}^{-\tau_{m}-1} \beta^{-j-1} \frac{1}{-j}\left(\sum_{i=k+j}^{k-1} \eta(i)\right)^{T} \\
\cdot & Z_{2}\left(\sum_{i=k+j}^{k-1} \eta^{T}(i)\right) \geq \frac{\tau_{M m}}{\tau_{M}} \\
& \cdot \sum_{j=-\tau_{M}}^{-\tau_{m}} \beta^{-j-1}\left(\begin{array}{c}
x(k) \\
x(k+j)
\end{array}\right)^{T}\left[\begin{array}{l}
Z_{2} \\
* \\
*
\end{array}\right]\left(\begin{array}{c}
x(k) \\
x(k+j)
\end{array}\right) \\
\geq & \frac{\tau_{M m}}{\tau_{M}} \sum_{i=k-\tau_{M}}^{k-\tau_{m}-1} \beta^{k-i-1}\left(\begin{array}{c}
x(k) \\
x(i)
\end{array}\right)^{T} \\
& \cdot\left[\begin{array}{l}
Z_{2} \\
* Z_{2} \\
*
\end{array} Z_{2}\right]\left(\begin{array}{l}
x(k) \\
x(i)
\end{array}\right) .
\end{aligned}
$$

Noticing that

$$
\sum_{i=k-\tau_{M}}^{k-1} \beta^{k-i-1}=\sum_{i=-\tau_{M}}^{-1} \beta^{-i-1}=\frac{1-\beta^{\tau_{M}}}{1-\beta}=\frac{1}{\delta}
$$

we have

$V_{1}(k)$

$$
=\delta x^{T}(k) P_{1} x(k)\left(\sum_{i=k-\tau_{M}}^{k-\tau_{m}-1} \beta^{k-i-1}+\sum_{i=k-\tau_{m}}^{k-1} \beta^{k-i-1}\right),
$$

$V_{2}(k)$

$$
\begin{gathered}
=\sum_{i=k-\tau_{m}}^{k-1} \beta^{k-1-i} x^{T}(i)\left(Q_{1}+Q_{2}\right) x(i) \\
+\sum_{i=k-\tau_{M}}^{k-\tau_{m}-1} \beta^{k-1-i} x^{T}(i) Q_{2} x(i) .
\end{gathered}
$$

It follows from (23)-(25) that

$$
\begin{aligned}
V(k) \geq & \sum_{i=k-\tau_{m}}^{k-1} \beta^{k-i-1}\left(\begin{array}{l}
x(k) \\
x(i)
\end{array}\right)^{T} \Upsilon_{1}\left(\begin{array}{l}
x(k) \\
x(i)
\end{array}\right) \\
& +\sum_{i=k-\tau_{M}}^{k-\tau_{m}-1} \beta^{k-i-1}\left(\begin{array}{c}
x(k) \\
x(i)
\end{array}\right)^{T} \Upsilon_{2}\left(\begin{array}{l}
x(k) \\
x(i)
\end{array}\right),
\end{aligned}
$$

where

$$
\begin{aligned}
& \Upsilon_{1}=\left[\begin{array}{cc}
\delta P_{1}+Z_{1} & -Z_{1} \\
* & Q_{1}+Q_{2}+Z_{1}
\end{array}\right], \\
& \Upsilon_{2}=\left[\begin{array}{cc}
\delta P_{1}+\frac{\tau_{M m}}{\tau_{M}} Z_{2} & -\frac{\tau_{M m}}{\tau_{M}} Z_{2} \\
* & Q_{2}+\frac{\tau_{M m}}{\tau_{M}} Z_{2}
\end{array}\right] .
\end{aligned}
$$

From condition (15), we have

$$
\Upsilon_{i}>\left[\begin{array}{cc}
\delta W & 0 \\
0 & 0
\end{array}\right], \quad i=1,2
$$

Together with (26)-(28), we can get

$$
\begin{aligned}
V(k) & \geq \delta x^{T}(k) W x(k) \sum_{i=k-\tau_{M}}^{k-1} \beta^{k-i-1}=x^{T}(k) W x(k) \\
& >0
\end{aligned}
$$

Then, taking the forward difference of $V(k)$ with respect to (1), we can get

$$
\begin{aligned}
& \Delta V_{1}(k)=(x(k)+\eta(k))^{T} P_{1}(x(k)+\eta(k)) \\
& \quad-\beta x^{T}(k) P_{1} x(k)-(1-\beta) V_{1}(k)=(1-\beta) x^{T}(k) \\
& \cdot P_{1} x(k)+2 x^{T}(k) P_{1} \eta(k)+\eta^{T}(k) P_{1} \eta(k)-(1 \\
& -\beta) V_{1}(k)
\end{aligned}
$$

$$
\Delta V_{2}(k)=\sum_{i=k-\tau_{m}+1}^{k} \beta^{k-i} x^{T}(i) Q_{1} x(i)-\sum_{i=k-\tau_{m}}^{k-1} \beta^{k-i} x^{T}(i)
$$

$$
\begin{aligned}
& \cdot Q_{1} x(i)+\sum_{i=k-\tau_{M}+1}^{k} \beta^{k-i} x^{T}(i) Q_{2} x(i) \\
& -\sum_{i=k-\tau_{M}}^{k-1} \beta^{k-i} x^{T}(i) Q_{2} x(i)-(1-\beta)
\end{aligned}
$$$$
\cdot \sum_{i=k-\tau_{m}}^{k-1} \beta^{k-i-1} x^{T}(i) Q_{1} x(i)-(1-\beta)
$$$$
\sum_{i=k-\tau_{M}}^{k-1} \beta^{k-i-1} x^{T}(i) Q_{2} x(i)=x^{T}(k) Q_{1} x(k)
$$$$
+x^{T}(k) Q_{2} x(k)-\beta^{\tau_{m}} x^{T}\left(k-\tau_{m}\right) Q_{1} x\left(k-\tau_{m}\right)
$$$$
-\beta^{\tau_{M}} x^{T}\left(k-\tau_{M}\right) Q_{2} x\left(k-\tau_{M}\right)-(1-\beta) V_{2}(k),
$$

$$
\Delta V_{3}(k)=\tau_{m} \sum_{i=-\tau_{m}}^{-1}\left[\eta^{T}(k) Z_{1} \eta(k)\right.
$$

$$
\begin{aligned}
& \left.-\beta^{-i} \eta^{T}(k+i) Z_{1} \eta(k+i)\right] \\
& +\tau_{M m} \sum_{i=-\tau_{M}}^{-\tau_{m}-1}\left[\eta^{T}(k) Z_{2} \eta(k)\right. \\
& \left.-\beta^{-i} \eta(k+i) Z_{2} \eta(k+i)\right]-(1-\beta) V_{3}(k) \\
& \leq \eta^{T}(k)\left(\tau_{m}^{2} Z_{1}+\tau_{M m}^{2} Z_{2}\right) \eta(k)
\end{aligned}
$$




$$
\begin{aligned}
& -\tau_{m} \beta^{\tau_{m}} \sum_{i=k-\tau_{m}}^{k-1} \eta^{T}(i) Z_{1} \eta(i)-\tau_{M m} \beta^{\tau_{M}} \sum_{i=k-\tau_{M}}^{k-\tau_{m}-1} \eta^{T}(i) \\
& \cdot Z_{2} \eta(i)-(1-\beta) V_{3}(k) .
\end{aligned}
$$

Based on Lemma 2, we have

$$
\begin{aligned}
& -\tau_{m} \sum_{i=k-\tau_{m}}^{k-1} \eta^{T}(i) Z_{1} \eta(i) \leq-\left[x(k)-x\left(k-\tau_{m}\right)\right]^{T} \\
& \cdot Z_{1}\left[x(k)-x\left(k-\tau_{m}\right)\right] \\
& -3\left[x(k)+x\left(k-\tau_{m}\right)-\kappa_{1}(k)\right]^{T} \\
& \cdot Z_{1}\left[x(k)+x\left(k-\tau_{m}\right)-\kappa_{1}(k)\right] .
\end{aligned}
$$

When $h_{m}<\tau(k)<h_{M}$, by using Lemma 2 , we obtain

$$
\begin{aligned}
& -\left(\tau_{M}-\tau_{m}\right) \sum_{i=k-\tau_{M}}^{k-\tau_{m}-1} \eta^{T}(i) Z_{2} \eta(i)=-\left(\tau_{M}-\tau_{m}\right) \\
& \quad \sum_{i=k-\tau(k)}^{k-\tau_{m}-1} \eta^{T}(i) Z_{2} \eta(i)-\left(\tau_{M}-\tau_{m}\right) \\
& \quad \sum_{i=k-\tau_{M}}^{k-\tau(k)-1} \eta^{T}(i) Z_{2} \eta(i) \\
& \quad \leq-\frac{\tau_{M m}}{\tau(k)-\tau_{m}}\left[\pi_{1}^{T}(k) Z_{2} \pi_{1}(k)\right. \\
& \left.+3 \pi_{2}^{T}(k) Z_{2} \pi_{2}(k)\right]-\frac{\tau_{M m}}{\tau_{M}-\tau(k)}\left[\pi_{3}^{T}(k) Z_{2} \pi_{3}(k)\right. \\
& \left.+3 \pi_{4}^{T}(k) Z_{2} \pi_{4}(k)\right]
\end{aligned}
$$

where $\pi_{1}(k)=x\left(k-\tau_{m}\right)-x(k-\tau(k)), \pi_{2}(k)=x\left(k-\tau_{m}\right)+$ $x(k-\tau(k))-\kappa_{2}(k), \pi_{3}(k)=x(k-\tau(k))-x\left(k-\tau_{M}\right)$, and $\pi_{4}(k)=x(k-\tau(k))+x\left(k-\tau_{M}\right)-\kappa_{3}(k)$.

Noting $\left(\tau(k)-\tau_{m}\right) / \tau_{M m}+\left(\tau_{M}-\tau(k)\right) / \tau_{M m}=1$ and $\left[\begin{array}{cc}\overline{Z_{2}} & s \\ * & \overline{Z_{2}}\end{array}\right]>0$ and using Lemma 3 , we have

$$
\begin{gathered}
-\left(\tau_{M}-\tau_{m}\right) \sum_{i=k-\tau_{M}}^{k-\tau_{m}-1} \eta^{T}(k) Z_{2} \eta(k) \\
\leq-\alpha^{T}(k)\left[\begin{array}{cc}
\overline{Z_{2}} & S \\
* & \overline{Z_{2}}
\end{array}\right] \alpha(k),
\end{gathered}
$$

where $\overline{Z_{2}}=\left[\begin{array}{cc}Z_{2} & 0 \\ 0 & 3 Z_{2}\end{array}\right]$ and $\alpha^{T}(k)=\left[\pi_{1}^{T}(k), \pi_{2}^{T}(k), \pi_{3}^{T}(k)\right.$, $\left.\pi_{4}^{T}(k)\right]$.

If $\tau(k)=\tau_{m}$, we have $x\left(k-\tau_{m}\right)-x(k-\tau(k))=0, x(k-$ $\left.\tau_{m}\right)+x(k-\tau(k))-\omega\left(k, \tau_{m}, \tau(k)\right)=0$; if $\tau(k)=\tau_{M}$, we also have $x(k-\tau(k))-x\left(k-\tau_{M}\right)=0, x(k-\tau(k))+x\left(k-\tau_{M}\right)-$ $\omega\left(k, \tau(k), \tau_{M}\right)=0$. Accordingly, when $\tau(k)=\tau_{m}$ or $\tau(k)=$ $\tau_{M},(33)$ still holds.
Thus, we obtain

$$
\begin{aligned}
\Delta V_{3}(k) \leq & \eta^{T}(k)\left(\tau_{m}^{2} Z_{1}+\left(\tau_{M m}\right)^{2} Z_{2}\right) \eta(k) \\
- & \beta^{\tau_{m}}\left[x(k)-x\left(k-\tau_{m}\right)\right]^{T} \\
& \cdot Z_{1}\left[x(k)-x\left(k-\tau_{m}\right)\right] \\
- & 3 \beta^{\tau_{m}}\left[x(k)+x\left(k-\tau_{m}\right)-\kappa_{1}(k)\right]^{T} \\
& \cdot Z_{1}\left[x(k)+x\left(k-\tau_{m}\right)-\kappa_{1}(k)\right] \\
- & \beta^{\tau_{M}} \alpha^{T}(k)\left[\begin{array}{cc}
Z_{2} & S \\
* & \overline{Z_{2}}
\end{array}\right] \alpha(k)-(1-\beta) \\
& \cdot V_{3}(k) .
\end{aligned}
$$

From (30)-(34), we have

$$
\begin{aligned}
& \Delta V(k)+(1-\beta) V(k)-\frac{(1-\beta) \omega^{T}(k) \omega(k)}{\omega_{d}^{2}} \\
& \quad \leq \zeta^{T}(k) \Xi_{1} \zeta(k),
\end{aligned}
$$

where $\zeta^{T}(k)=\left[x^{T}(k), x^{T}\left(k-\tau_{m}\right), x^{T}(k-\tau(k)), x^{T}(k-\right.$ $\left.\left.\tau_{M}\right), \kappa_{1}^{T}(k), \kappa_{2}^{T}(k), \kappa_{3}^{T}(k), \eta^{T}(k), \omega^{T}(k)\right]$.

For any matrices $M_{1}, M_{2}, M_{3}$ with appropriate dimensions, we have

$$
\begin{gathered}
2\left[x^{T}(k) M_{1}+x^{T}(k-\tau(k)) M_{2}+\eta^{T}(k) M_{3}\right] \\
\cdot\left[\left(A_{1}-I\right) x(k)+A_{2} x(k-\tau(k))+B \omega(k)\right. \\
-\eta(k)]=\zeta^{T}(k) \Xi_{2} \zeta(k)=0 .
\end{gathered}
$$

From (35)-(36), we can get

$$
\begin{aligned}
& \Delta V(k)+(1-\beta) V(k)-\frac{(1-\beta) \omega^{T}(k) \omega(k)}{\omega_{d}{ }^{2}} \\
& \quad \leq \zeta^{T}(k)\left(\Xi_{1}+\Xi_{2}\right) \zeta(k) .
\end{aligned}
$$

Noting that $\Xi=\Xi_{1}+\Xi_{2}<0$, we obtain

$$
\Delta V(k)+(1-\beta) V(k)-\frac{(1-\beta) \omega^{T}(k) \omega(k)}{\omega_{d}^{2}} \leq 0 .
$$

Furthermore, we describe the initial value of Lyapunov functional as

$$
\begin{aligned}
& V(0)=x^{T}(0) P_{1} x(0)+\sum_{i=-\tau_{m}}^{-1} \beta^{-1-i} x^{T}(i) Q_{1} x(i) \\
& +\sum_{i=-\tau_{M}}^{-1} \beta^{-1-i} x^{T}(i) Q_{2} x(i) \\
& +\sum_{j=-\tau_{m}}^{-1} \sum_{i=j}^{-1} \beta^{-1-i} \eta^{T}(i) Z_{1} \eta(i)
\end{aligned}
$$




$$
\begin{aligned}
& +\sum_{j=-\tau_{M}}^{-\tau_{m}-1} \sum_{i=j}^{-1} \beta^{-1-i} \eta^{T}(i) Z_{2} \eta(i) \leq\left|\lambda_{1}\right| x^{T}(0) x(0) \\
& +\left|\lambda_{2}\right| \sum_{i=-\tau_{m}}^{-1} x^{T}(i) x(i)+\left|\lambda_{3}\right| \sum_{i=-\tau_{M}}^{-1} x^{T}(i) x(i) \\
& +\lambda_{4} \sum_{j=-\tau_{m}}^{-1} \sum_{i=j}^{-1} \eta^{T}(i) \eta(i)+\beta^{h_{m}} \lambda_{5} \sum_{j=-\tau_{M}}^{-h_{m}-1} \sum_{i=j}^{-1} \eta^{T}(i) \eta(i) \\
& =\left(\left|\lambda_{1}\right|+\tau_{m}\left|\lambda_{2}\right|+\tau_{M}\left|\lambda_{3}\right|\right) \sigma_{1}+\left(\frac{\tau_{m}\left(\tau_{m}-1\right)}{2} \lambda_{4}\right. \\
& \left.+\beta^{\tau_{m}} \frac{\left(\tau_{M}-\tau_{m}\right)\left(\tau_{M}+\tau_{m}+1\right)}{2} \lambda_{5}\right) \sigma_{2}=\mu_{1} \sigma_{1} \\
& +\mu_{2} \sigma_{2} .
\end{aligned}
$$

By condition (17) and Lemma 1, we have $V(k) \leq 1$. Then, together with (15), it is implied that $x^{T}(k) W x(k) \leq 1$. Thus, this means that the reachable set of system (1) is contained in the ellipsoid defined by (19). This completes the proof.

Remark 5. The difficulty of this paper is how to handle the term $\sum_{j=-\tau_{m}}^{-1} \sum_{i=k+j}^{k-1} \eta^{T}(i) Z_{1} \eta(i)$ and the term $\sum_{j=-\tau_{M}}^{-\tau_{m}-1} \sum_{i=k+j}^{k-1} \eta^{T}(i) Z_{2} \eta(i)$. In this paper, we using discrete Wirtinger-based inequality instead of discrete Jensen inequality [38-40] to deal with the term $\sum_{j=-\tau_{m}}^{-1} \sum_{i=k+j}^{k-1} \eta^{T}(i) Z_{1} \eta(i)$. Then, by using reciprocally convex approach and discrete Wirtinger-based inequality, the new inequality (33) is introduced to deal with the term $\sum_{j=-\tau_{M}}^{-\tau_{m}-1} \sum_{i=k+j}^{k-1} \eta^{T}(i) Z_{2} \eta(i)$.

Remark 6. It is worth pointing out that our results are more relaxed and general compared with the results in [38], because the initial condition is not required to be zero in system (1). In the case where the initial set contains only the origin point, that is, $\sigma_{1}=\sigma_{2}=0$, it is easy to get $V(0)=0$ without needing (17). Therefore, we can obtain reachable set bounding for discrete-time system with time-varying delay and it is stated in the following theorem.

Theorem 7. For given scalars $0<\beta<1$, if there exist symmetric matrices $P_{1} \in \mathbb{R}^{n \times n}, Q_{1} \in \mathbb{R}^{n \times n}, Q_{2} \in \mathbb{R}^{n \times n}$, $Z_{1} \in \mathbb{R}^{n \times n}>0, Z_{2} \in \mathbb{R}^{n \times n}>0, W \in \mathbb{R}^{n \times n}>0$ and matrices $S \in \mathbb{R}^{2 n \times 2 n}, M_{1} \in \mathbb{R}^{n \times n}, M_{2} \in \mathbb{R}^{n \times n}, M_{3} \in \mathbb{R}^{n \times n}$, such that

$$
\begin{aligned}
\Phi_{1} & =\left[\begin{array}{ll}
\overline{Z_{2}} & S \\
* & \overline{Z_{2}}
\end{array}\right]>0, \\
\Omega_{1} & >0, \\
\Omega_{2} & >0, \\
\Xi & =\Xi_{1}+\Xi_{2}<0,
\end{aligned}
$$

then, the reachable set of system (1) is contained in the following ellipsoid:

$$
\varepsilon(W)=\left\{\xi \in \mathbb{R}^{n} \mid \xi^{T} W \xi \leq 1\right\},
$$

where $\Xi_{1}, \Xi_{2}, \Omega_{1}, \Omega_{2}$ are defined in Theorem 4 .

Remark 8. Traditionally, it was always assumed that each of the involved symmetric matrices must be positive, which ensured that the corresponding Lyapunov-Krasovskii functional would be positive, while, in this paper, we relaxed these conditions. Particularly, we established a novel LyapunovKrasovskii functional that the involved matrices such as $P$, $Q_{1}$, and $Q_{2}$ may be nonpositive.

Remark 9. The difficulty of this method lies in how to prove that the relevant Lyapunov-Krasovskii functional $V(k)$ must be positive definite. In order to overcome this difficulty, we take full advantage of Jensen's inequality; then, one can obtain the conditions (15), which ensure $V(k) \geq x^{T}(k) W x(k)>0$. If the methods in [38] are followed, all the involved symmetric matrices in Lyapunov-Krasovskii functional being positive definite implies that $V(k) \geq x^{T}(k) P x(k)>0$. We can easily obtain the following corollaries.

Corollary 10. For given scalars $0<\beta<1$, if there exist positive scalars $\lambda_{1}, \lambda_{2}, \lambda_{3}, \lambda_{4}, \lambda_{5}$, symmetric matrices $P_{1} \in$ $\mathbb{R}^{n \times n}>0, Q_{1} \in \mathbb{R}^{n \times n}>0, Q_{2} \in \mathbb{R}^{n \times n}>0, Z_{1} \in \mathbb{R}^{n \times n}>0$, $Z_{2} \in \mathbb{R}^{n \times n}>0$, and matrices $S \in \mathbb{R}^{2 n \times 2 n}, M_{1} \in \mathbb{R}^{n \times n}$, $M_{2} \in \mathbb{R}^{n \times n}, M_{3} \in \mathbb{R}^{n \times n}$, such that

$$
\begin{aligned}
\Phi_{1} & =\left[\begin{array}{ll}
\overline{Z_{2}} & S \\
* & \overline{Z_{2}}
\end{array}\right]>0, \\
P_{1} & \leq \lambda_{1} I, \\
Q_{1} & \leq \lambda_{2} I, \\
Q_{2} & \leq \lambda_{3} I, \\
0 & <Z_{1} \leq \lambda_{4} I, \\
0 & <Z_{2} \leq \lambda_{5} I, \\
\mu_{1} \sigma_{1}+\mu_{2} \sigma_{2} & \leq 1, \\
\Xi & =\Xi_{1}+\Xi_{2}<0,
\end{aligned}
$$

then, the reachable set of system (1) is contained in the following ellipsoid:

$$
\varepsilon\left(P_{1}\right)=\left\{\xi \in \mathbb{R}^{n} \mid \xi^{T} P_{1} \xi \leq 1\right\},
$$

where $\Xi_{1}, \Xi_{2}, \mu_{1}, \mu_{2}$ are defined in Theorem 4.

Corollary 11. For given scalars $0<\beta<1$, if there exist symmetric matrices $P_{1} \in \mathbb{R}^{n \times n}>0, Q_{1} \in \mathbb{R}^{n \times n}>0$, $Q_{2} \in \mathbb{R}^{n \times n}>0, Z_{1} \in \mathbb{R}^{n \times n}>0, Z_{2} \in \mathbb{R}^{n \times n}>0$, and matrices $S \in \mathbb{R}^{2 n \times 2 n}, M_{1} \in \mathbb{R}^{n \times n}, M_{2} \in \mathbb{R}^{n \times n}, M_{3} \in \mathbb{R}^{n \times n}$, such that

$$
\begin{aligned}
\Phi_{1} & =\left[\begin{array}{cc}
\overline{Z_{2}} & S \\
* & \overline{Z_{2}}
\end{array}\right]>0, \\
\Xi & =\Xi_{1}+\Xi_{2}<0,
\end{aligned}
$$


then, the reachable set of system (1) is contained in the following ellipsoid:

$$
\varepsilon\left(P_{1}\right)=\left\{\xi \in \mathbb{R}^{n} \mid \xi^{T} P_{1} \xi \leq 1\right\},
$$

where $\Xi_{1}, \Xi_{2}$ are defined in Theorem 4 .

Remark 12. To get the smallest possible ellipsoid with the shortest major axis, we consider the matrix $W$ as a decision variable and satisfying $0<\gamma I \leq W$, which is equivalent to

$$
\left[\begin{array}{cc}
-\bar{\gamma} I & I \\
I & -W
\end{array}\right] \leq 0
$$

where $\bar{\gamma}=1 / \gamma$. Then the smallest possible ellipsoid can be obtained when $\bar{\delta}$ is minimum.

Remark 13. In order to solve matrix inequalities (14)-(18) with 6 parameters $\lambda_{1}, \lambda_{2}, \lambda_{3}, \lambda_{4}, \lambda_{5}$, and $\beta$, we first simplify them into linear matrix inequalities (LMIs). For example, matrix inequality $P_{1} \leq \lambda_{1}$ is represented as linear matrix inequality $P_{1} \leq \widetilde{P}_{1}$, where $\widetilde{P}_{1}=\operatorname{diag}\left\{\lambda_{1}, \lambda_{1}, \ldots, \lambda_{1}\right\}$. Similarly, matrix inequalities (16) are also represented as LMIs. Then, by fixing parameter $\beta$, matrix inequalities (15), (17), and (18) are reduced to LMIs. Thus, to compute the smallest bound of a reachable set for discrete-time systems (1), we solve the optimization problem for a parameter $\gamma>0$ :

$$
\begin{array}{ll}
\min & \bar{\gamma} \quad\left(\bar{\gamma}=\frac{1}{\gamma}\right) \\
\text { s.t. } & \begin{cases}(a) & \gamma I \leq W \\
(b) & (14)-(18) .\end{cases}
\end{array}
$$

\section{Numerical Examples}

In this section, we present two examples to demonstrate the effectiveness of our results.

Example 14. Consider the discrete-time system (1) with the following parameters:

$$
\begin{aligned}
A_{1} & =\left[\begin{array}{cc}
0.8 & -0.01 \\
-0.5 & 0.09
\end{array}\right], \\
A_{2} & =\left[\begin{array}{cc}
-0.02 & 0 \\
-0.1 & -0.01
\end{array}\right], \\
B & =\left[\begin{array}{l}
0.2 \\
0.1
\end{array}\right],
\end{aligned}
$$

and $\omega_{d}=0.1$ and $\delta_{1}=\delta_{2}=0$. The time-varying delay satisfied $2 \leq \tau(k) \leq 6$, by applying Theorem 7 with the Matlab LMI tool box, the minimal $\bar{\gamma}=2.2594$ is obtained when $\beta=0.7$, and some of the obtained decision variables are

$$
\begin{aligned}
& W=\left[\begin{array}{ll}
1.3013 & 0.2415 \\
0.2415 & 0.5105
\end{array}\right], \\
& Q_{1}=\left[\begin{array}{cc}
-0.2262 & -0.0527 \\
-0.0527 & 0.2064
\end{array}\right] \text {, } \\
& Q_{2}=\left[\begin{array}{cc}
0.2974 & -0.0665 \\
-0.0665 & 0.6575
\end{array}\right] \text {, } \\
& Z_{1}=\left[\begin{array}{cc}
0.8662 & -0.1165 \\
-0.1165 & 0.1029
\end{array}\right] \text {, } \\
& Z_{2}=\left[\begin{array}{cc}
0.6042 & -0.0819 \\
-0.0819 & 0.1321
\end{array}\right] \text {, } \\
& S_{11}=\left[\begin{array}{ll}
0.2372 & -0.0351 \\
0.0403 & -0.0927
\end{array}\right] \text {, } \\
& S_{12}=\left[\begin{array}{cc}
0.0118 & 0.0006 \\
-0.0023 & 0.0029
\end{array}\right] \text {, } \\
& S_{21}=\left[\begin{array}{ll}
-0.0286 & 0.0023 \\
-0.0047 & 0.0004
\end{array}\right] \text {, } \\
& S_{22}=\left[\begin{array}{ll}
0.0623 & -0.0004 \\
0.0206 & -0.0044
\end{array}\right] \text {, } \\
& M_{1}=\left[\begin{array}{cc}
2.0413 & -0.0402 \\
1.6849 & 2.0052
\end{array}\right] \text {, } \\
& M_{2}=\left[\begin{array}{cc}
-0.1119 & -0.1517 \\
0.0906 & -0.2828
\end{array}\right] \text {, } \\
& M_{3}=\left[\begin{array}{cc}
13.3680 & -1.1525 \\
-3.7618 & 5.0743
\end{array}\right] \text {. }
\end{aligned}
$$

The eigenvalues of $Q_{1}$ are -0.2325 and 0.2127 . Obviously, the matrix $Q_{1}$ is not positive definite. Hence, there really exist nonpositive-definite matrices $Q_{1}$ such that the LKF in (21) is positive definite. Moreover, by the method in [40], the minimal $\bar{\gamma}=2.8160$ is obtained. Figure 1 gives a more intuitive comparison of the reachable sets by different methods; it is clear that our method in this paper yields much tighter bounds than that of [40]. Therefore, it can be seen that our results are less conservative than the ones in [40]. Figure 2 shows the reachable set when $2 \leq \tau(k) \leq 8$ and $2 \leq \tau(k) \leq 10$; from Figure 2, we can see that the reachable set of the system is related to the time delay.

Example 15. Consider the discrete-time system (1) with the following parameters:

$$
A_{1}=\left[\begin{array}{cc}
0.8 & 0.1 \\
0 & 0.97
\end{array}\right],
$$




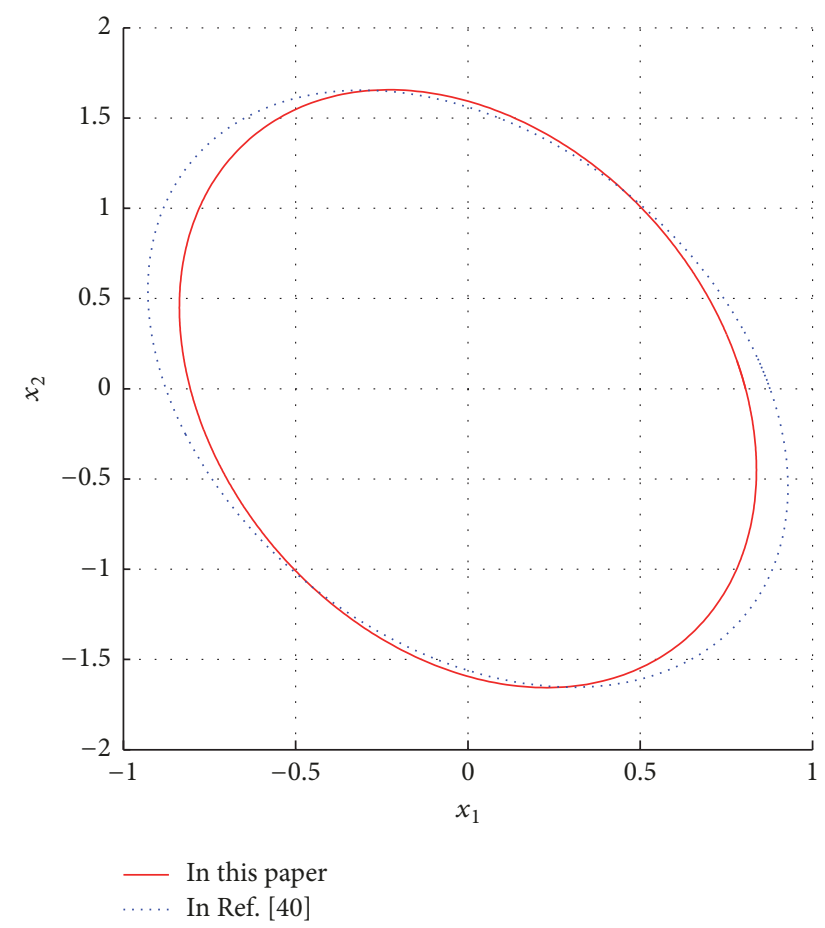

Figure 1: The bounding reachable set computed by different methods.

TABLE 1: Different values of $\tau_{M}$ with different $\tau_{m}$.

\begin{tabular}{lcc}
\hline$\tau_{m}$ & 4 & 5 \\
\hline Theorem 1, [38] & 7 & 7 \\
Theorem 1, [40] & 14 & 11 \\
Corollary 11 & 16 & 15 \\
\hline
\end{tabular}

$$
\begin{aligned}
A_{2} & =\left[\begin{array}{cc}
-0.1 & 0 \\
-0.1 & -0.1
\end{array}\right], \\
B & =\left[\begin{array}{l}
0.1 \\
0.1
\end{array}\right],
\end{aligned}
$$

and $\omega_{d}=0.1$ and $\delta_{1}=\delta_{2}=0$. The system has been considered in some existing works $[38,40]$. The allowable maximal values of $\tau_{M}$ are listed in Table 1 with different $\tau_{m}$. Therefore, our conditions proposed in Corollary 11 provide a better range for the time-varying delay as shown in Table 1.

\section{Conclusions}

In this paper, the problem of reachable set bounding for discrete-time system with time-varying delay and bounded disturbance inputs has been investigated, by establishing a novel Lyapunov-Krasovskii functional, which does not require all the involved symmetric matrices to be positive definite. Then, we present some new criteria based on discrete Wirtinger-based inequality and reciprocally convex

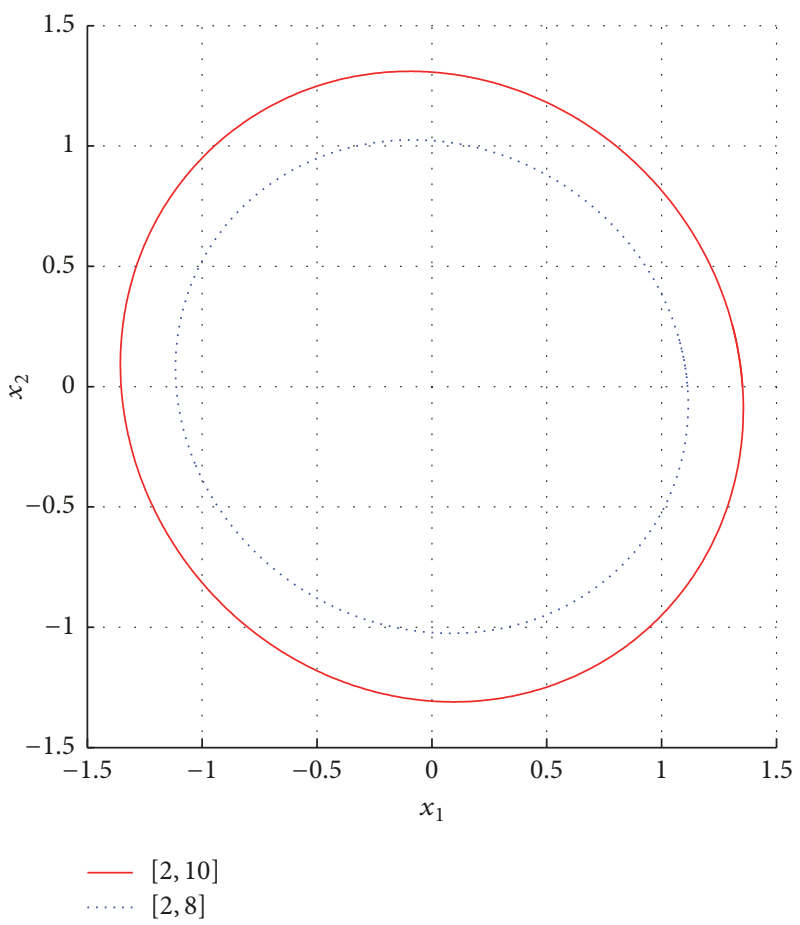

FIGURE 2: The bounding reachable set computed by different timevarying delay.

method. Two numerical examples are provided to validate the effectiveness of the proposed methods.

\section{Conflicts of Interest}

The authors declare that they have no conflicts of interest.

\section{Acknowledgments}

This study is supported by the Natural Science Foundation of the Anhui Higher Education Institutions of China (KJ2016A555, KJ2016A625), the Program for Innovative Research Team of the Higher Education Institutions of Hubei Province (T201812), the Natural Science Foundation of the Hubei Province of China (2016CFB211), Program for Excellent Young Talents in University of Anhui Province (gxyq2017158), and Provincial Natural Science Foundation of Anhui (1708085MF155).

\section{References}

[1] J. Abedor, K. Nagpal, and K. Poolla, "A linear matrix inequality approach to peak-to-peak gain minimization," International Journal of Robust and Nonlinear Control, vol. 6, no. 9-10, pp. 899-927, 1996.

[2] E. Fridman and U. Shaked, "On reachable sets for linear systems with delay and bounded peak inputs," Automatica, vol. 39, no. 11, pp. 2005-2010, 2003.

[3] J.-H. Kim, "Improved ellipsoidal bound of reachable sets for time-delayed linear systems with disturbances," Automatica, vol. 44, no. 11, pp. 2940-2943, 2008. 
[4] A. Bellen, N. Guglielmi, and A. E. Ruehli, "Methods for linear systems of circuit delay differential equations of neutral type," IEEE Transactions on Circuits and Systems I: Fundamental Theory and Applications, vol. 46, no. 1, pp. 212-216, 1999.

[5] B. Wang, J. Yan, J. Cheng, and S. Zhong, "New criteria of stability analysis for generalized neural networks subject to time-varying delayed signals," Applied Mathematics and Computation, vol. 314, pp. 322-333, 2017.

[6] Y. Liu, W. Ma, M. S. Mahmoud, and S. . Lee, "Improved delay-dependent exponential stability criteria for neutral-delay systems with nonlinear uncertainties," Applied Mathematical Modelling: Simulation and Computation for Engineering and Environmental Systems, vol. 39, no. 10-11, pp. 3164-3174, 2015.

[7] F. Li, X. Meng, and Y. Cui, "Nonlinear stochastic analysis for a stochastic SIS epidemic model," Journal of Nonlinear Sciences and Applications, vol. 10, no. 9, pp. 5116-5124, 2017.

[8] Z. Wu, P. Shi, H. Su, and J. Chu, "Stability and dissipativity analysis of static neural networks with time delay," IEEE Transactions on Neural Networks and Learning Systems, vol. 23, no. 2, pp. 199-210, 2012.

[9] O. M. Kwon, M. J. Park, J. H. Park, S. M. Lee, and E. J. Cha, "New criteria on delay-dependent stability for discrete-time neural networks with time-varying delays," Neurocomputing, vol. 121, pp. 185-194, 2013.

[10] S. Zhu, Z. Li, and C. Zhang, "Delay decomposition approach to delay-dependent stability for singular time-delay systems," IET Control Theory \& Applications, vol. 4, no. 11, pp. 2613-2620, 2010.

[11] O. M. Kwon, M. J. Park, J. H. Park, S. M. Lee, and E. J. Cha, "New delay-partitioning approaches to stability criteria for uncertain neutral systems with time-varying delays," Journal of The Franklin Institute, vol. 349, no. 9, pp. 2799-2823, 2012.

[12] L. V. Hien and V. N. Phat, "Exponential stability and stabilization of a class of uncertain linear time-delay systems," Journal of The Franklin Institute, vol. 346, no. 6, pp. 611-625, 2009.

[13] P. Park, J. W. Ko, and C. Jeong, "Reciprocally convex approach to stability of systems with time-varying delays," Automatica, vol. 47, no. 1, pp. 235-238, 2011.

[14] Y. He, Q.-G. Wang, L. Xie, and C. Lin, "Further improvement of free-weighting matrices technique for systems with timevarying delay," Institute of Electrical and Electronics Engineers Transactions on Automatic Control, vol. 52, no. 2, pp. 293-299, 2007.

[15] A. Seuret and F. Gouaisbaut, "Wirtinger-based integral inequality: application to time-delay systems," Automatica, vol. 49, no. 9, pp. 2860-2866, 2013.

[16] M. Park, O. Kwon, J. H. Park, S. Lee, and E. Cha, "Stability of time-delay systems via Wirtinger-based double integral inequality," Automatica, vol. 55, pp. 204-208, 2015.

[17] P. T. Nam, P. N. Pathirana, and H. Trinh, "Discrete Wirtingerbased inequality and its application," Journal of The Franklin Institute, vol. 352, no. 5, pp. 1893-1905, 2015.

[18] H. Trinh, P. T. Nam, P. N. Pathirana, and H. . Le, "On backwards and forwards reachable sets bounding for perturbed time-delay systems," Applied Mathematics and Computation, vol. 269, pp. 664-673, 2015.

[19] I. M. Mitchell and C. J. Tomlin, "Overapproximating reachable sets by Hamilton-Jacobi projections," Journal of Scientific Computing, vol. 19, no. 1-3, pp. 323-346, 2003.

[20] P. T. Nam, P. N. Pathirana, and H. Trinh, "Partial state bounding with a pre-specified time of non-linear discrete systems with time-varying delays," IET Control Theory \& Applications, vol. 10, no. 13, pp. 1496-1502, 2016.
[21] P. T. Nam and P. N. Pathirana, "Further result on reachable set bounding for linear uncertain polytopic systems with interval time-varying delays," Automatica, vol. 47, no. 8, pp. 1838-1841, 2011.

[22] Z. Zuo, D. W. Ho, and Y. Wang, "Reachable set bounding for delayed systems with polytopic uncertainties: the maximal Lyapunov-Krasovskii functional approach," Automatica, vol. 46, no. 5, pp. 949-952, 2010.

[23] Z. Zuo, Z. Wang, Y. Chen, and Y. Wang, "A non-ellipsoidal reachable set estimation for uncertain neural networks with time-varying delay," Communications in Nonlinear Science and Numerical Simulation, vol. 19, no. 4, pp. 1097-1106, 2014.

[24] Z. Zuo, Y. Chen, Y. Wang, D. W. Ho, M. Z. Chen, and H. Li, "A note on reachable set bounding for delayed systems with polytopic uncertainties," Journal of The Franklin Institute, vol. 350, no. 7, pp. 1827-1835, 2013.

[25] Z. Feng and J. Lam, "An improved result on reachable set estimation and synthesis of time-delay systems," Applied Mathematics and Computation, vol. 249, pp. 89-97, 2014.

[26] O. M. Kwon, S. M. Lee, and J. H. Park, "On the reachable set bounding of uncertain dynamic systems with time-varying delays and disturbances," Information Sciences, vol. 181, no. 17, pp. 3735-3748, 2011.

[27] L. V. Hien and H. M. Trinh, "A new approach to state bounding for linear time-varying systems with delay and bounded disturbances," Automatica, vol. 50, no. 6, pp. 1735-1738, 2014.

[28] Z. Zuo, Y. Fu, and Y. Wang, "Results on reachable set estimation for linear systems with both discrete and distributed delays," IET Control Theory \& Applications, vol. 6, no. 14, pp. 2346-2350, 2012.

[29] Z. Zuo, D. W. Ho, and Y. Wang, "Reachable set estimation for linear systems in the presence of both discrete and distributed delays," IET Control Theory \& Applications, vol. 5, no. 15, pp. 1808-1812, 2011.

[30] C. Shen and S. Zhong, "The ellipsoidal bound of reachable sets for linear neutral systems with disturbances," Journal of The Franklin Institute, vol. 348, no. 9, pp. 2570-2585, 2011.

[31] Z. Feng and J. Lam, "On reachable set estimation of singular systems," Automatica, vol. 52, pp. 146-153, 2015.

[32] P. T. Nam, P. N. Pathirana, and H. Trinh, "Reachable set bounding for nonlinear perturbed time-delay systems: the smallest bound," Applied Mathematics Letters, vol. 43, pp. 6871, 2015.

[33] N. Zhang, Y. Sun, and P. Zhao, "State bounding for homogeneous positive systems of degree one with time-varying delay and exogenous input," Journal of The Franklin Institute, vol. 354, no. 7, pp. 2893-2904, 2017.

[34] Y. Zhang, P. Shi, S. K. Nguang, and Y. Song, "Robust finite-time $\mathrm{H}_{\infty}$ control for uncertain discrete-time singular systems with Markovian jumps," IET Control Theory \& Applications, vol. 8, no. 12, pp. 1105-1111, 2014.

[35] F. Amato and M. Ariola, "Finite-time control of discrete-time linear systems," Institute of Electrical and Electronics Engineers Transactions on Automatic Control, vol. 50, no. 5, pp. 724-729, 2005.

[36] W. Kang, S. Zhong, K. Shi, and J. Cheng, "Finite-time stability for discrete-time system with time-varying delay and nonlinear perturbations," ISA Transactions, vol. 60, pp. 67-73, 2016.

[37] W. Kang, S. Zhong, and J. Cheng, "Relaxed passivity conditions for discrete-time stochastic delayed neural networks," International Journal of Machine Learning and Cybernetics, vol. 7, no. 2, pp. 205-216, 2016. 
[38] N. D. That, P. T. Nam, and Q. P. Ha, "Reachable set bounding for linear discrete-time systems with delays and bounded disturbances," Journal of Optimization Theory and Applications, vol. 157, no. 1, pp. 96-107, 2013.

[39] J. Lam, B. Zhang, Y. Chen, and S. Xu, "Reachable set estimation for discrete-time linear systems with time delays," International Journal of Robust and Nonlinear Control, vol. 25, no. 2, pp. 269281, 2015.

[40] L. V. Hien, N. T. An, and H. Trinh, "New results on state bounding for discrete-time systems with interval time-varying delay and bounded disturbance inputs," IET Control Theory \& Applications, vol. 8, no. 14, pp. 1405-1414, 2014.

[41] Z. Feng, W. X. Zheng, and L. Wu, "Reachable set estimation of T-S fuzzy systems with time-varying delay," IEEE Transactions on Fuzzy Systems, vol. 25, no. 4, pp. 878-891, 2017.

[42] Z. Xu, H. Su, P. Shi, R. Lu, and Z.-G. Wu, "Reachable Set Estimation for Markovian Jump Neural Networks with TimeVarying Delays," IEEE Transactions on Cybernetics, vol. 47, no. 10, pp. 3208-3217, 2017. 


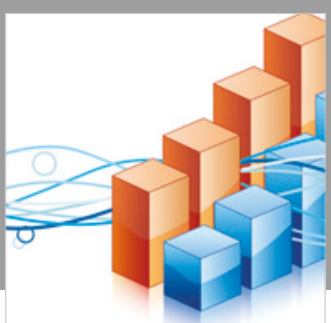

Advances in

Operations Research

\section{-n-m}
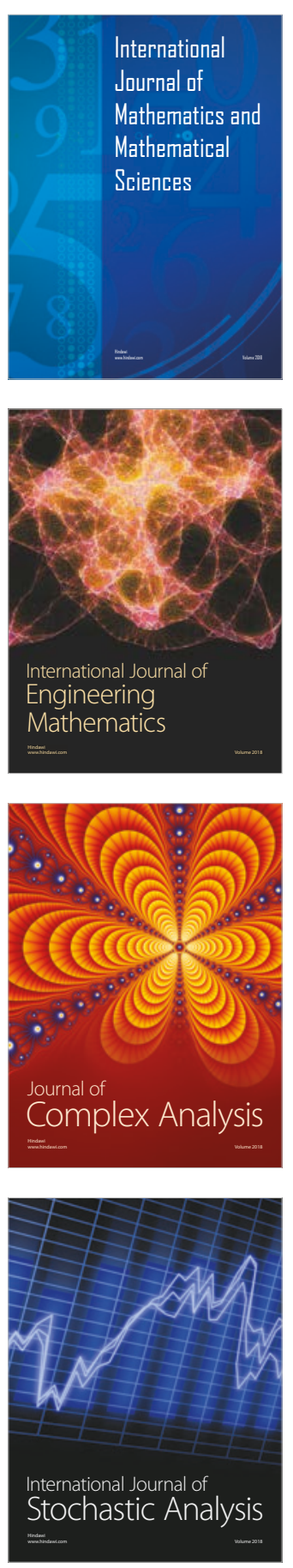
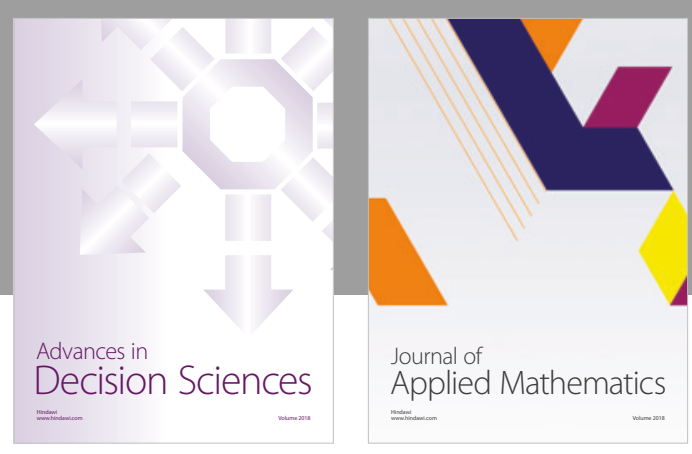

Journal of

Applied Mathematics
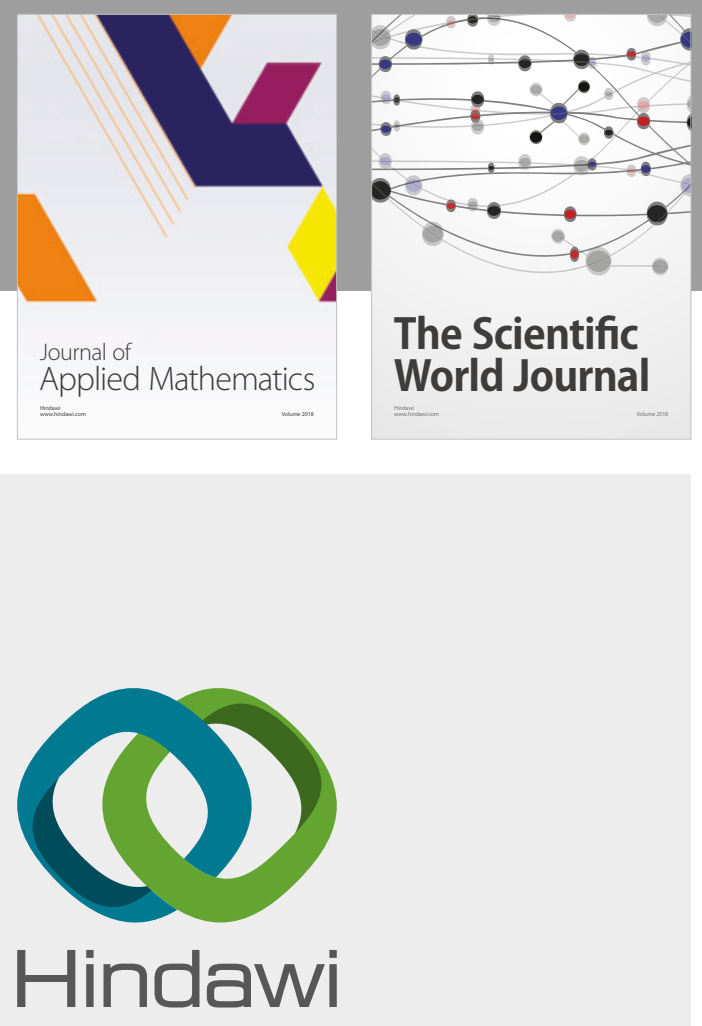

Submit your manuscripts at

www.hindawi.com

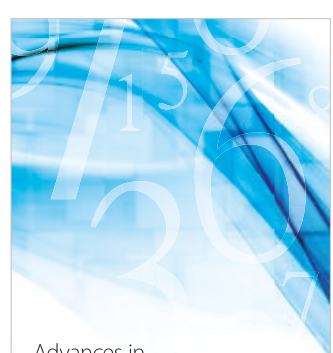

Advances in
Numerical Analysis
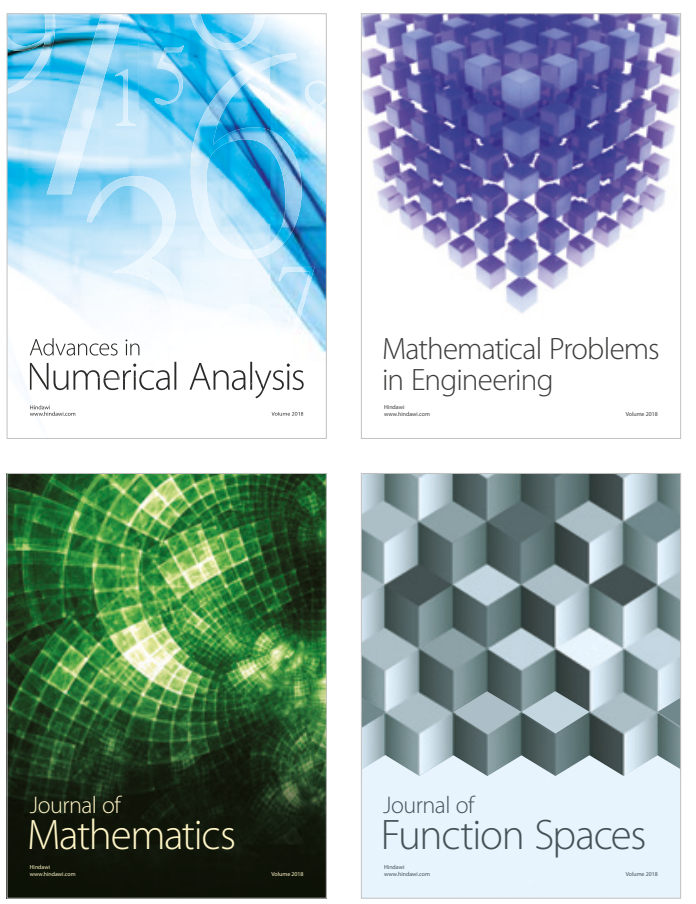

Mathematical Problems in Engineering

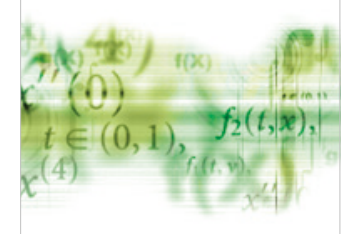

International Journal of

Differential Equations

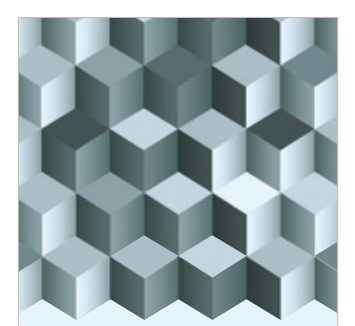

Journal of

Function Spaces
The Scientific

World Journal

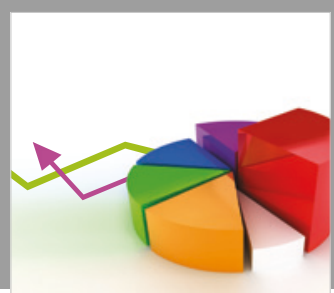

Journal of

Probability and Statistics
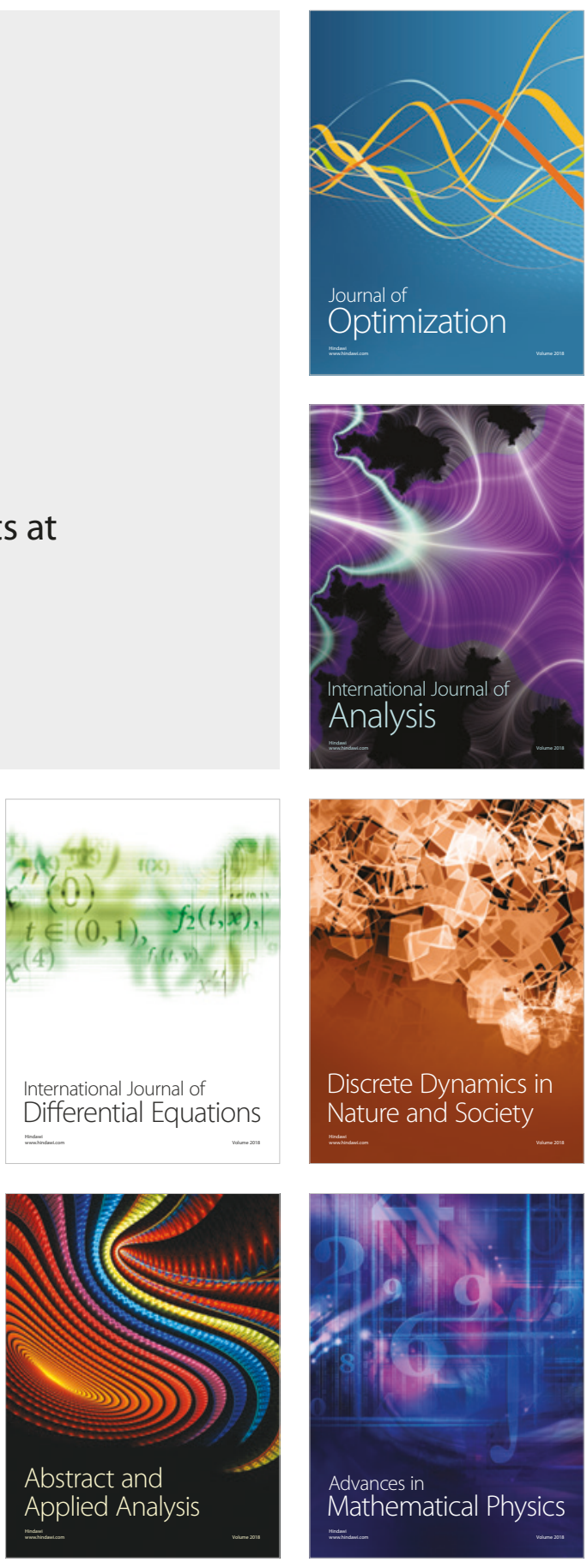